\title{
An experimental and simulation study on static tire enveloping stiffness
}

\author{
Chao-Zhi QIU*, Peng-Fei SUN***, Shui-Ting ZHOU*, Hong-Wu HUANG**** and Meng DU* \\ ${ }^{*}$ Collaborative Innovation Center for R\&D of Coach and Special Vehicle, Xiamen University of Technology \\ 361024 Xiamen, Fujian, China \\ E-mail: zhoushuiting@xmut.edu.cn \\ ${ }^{* *}$ School of Aerospace Engineering, Xiamen University, Xiamen 361005, China
}

Received: 17 February 2020; Revised: 14 April 2020; Accepted: 25 May 2020

\begin{abstract}
Three bump sizes, six pressures and five placement angles of bump were used in this paper to conduct enveloping stiffness experiments. The experimental tire displacement-load curves showed that the enveloping stiffness of the tire under the same pressure did not change with changes of the bump size. And the enveloping stiffness of the tire were changed with changes of pressures and placement angles; Models consider pressure and placement angle of bump were established respectively, and established a model consider pressure and placement angle simultaneously by least squares, those models can be used to estimate enveloping stiffness force without experiments. Abaqus finite element software simulations showed that the stress distribution in the tread is $\mathrm{H}$-shaped and the deformation distribution in the tread is oval; By analyzing the force distribution of the ply found that the distribution of force changes with the change of placement angles. The lateral displacement(U1) of the belts increases linearly with increasing angle. The end of cord is prone to failure at $0^{\circ}$ and $45^{\circ}$, and the cord near middle of ply is also prone to failure at all angles.
\end{abstract}

Keywords : Enveloping stiffness, Bump, Tire pressure, Placement angle, Abaqus, Least squares

\section{Introduction}

Tires are the only component of a car in contact with the ground, and the mechanical performance of the tire directly affects the comprehensive performance in terms of the car's handling stability and comfort. Enhancing the mechanical properties of tires can greatly improve the safety and stability of the vehicle. Tire stiffness is an indispensable part of the mechanical analysis of tires, and it is divided into lateral stiffness, longitudinal stiffness, torsional stiffness, radial stiffness, and enveloping stiffness. The enveloping stiffness reflects the tolerance of the tire to uneven surfaces, stones, and potholes. The enveloping stiffness of a tire will directly affect the comfort of the vehicle on the road.

Many studies (Zhou et al.,2009) have been performed on the enveloping stiffness with small bumps and quasi-static conditions used finite element software to simulate the stiffness characteristics of a tire, the bump width was $10 \mathrm{~mm}$, and the pressure was $0.2 \mathrm{MPa}$. The deformation contour and displacement load curves of the tire were obtained. The relationship between the rise heights of the bumps and load was basically consistent with the experimental values. Some studies ( $\mathrm{Li}$ et al.,2019) based on the static enveloping characteristics of tires used finite element software to simulate the rolling conditions of tires under different tire pressures and loads and low-speed rolling over a bulge. They found that at low speeds, the tire pressure and tire enveloping stiffness had a significant influence on the dynamic enveloping characteristics of the tire. Furthermore, the model that best matched the experimental data was composed of beam elements with a softening basis. The model was used to calculate the radial stiffness of the tire instead of the sidewall stiffness and inflation pressure, after which a model of the bump and tread was added using finite element software to study the effect of the load and radial stiffness on the enveloping characteristics of the tire. Some authors (Qiu et al.,1994) assumed that the radial stiffness of the tire was nonlinear, and 
the nonlinear relationship between the elastic force of the tire and the deformation of the tire was established using various methods. The sidewall stiffness was related to sidewall bending geometric characteristics and inflation pressure. Gent et al.(2013) discussed the calculation of the tire displacement-load and the stiffness relationship and found that the tire pressure was the main component of elasticity. Kang et al.(2009) accounted for the enveloping characteristics of the tire when predicting the natural frequency of the tire and analyzing the tire modality, and it was found that the natural frequency and mode of the tire were sensitive to changes in the tire stiffness and tire pressure, and used (Alkana and Karamihas,2011) three different bump shapes and different tire gauges to conduct enveloping stiffness experiments and found that the shape of the bump had little effect on tire enveloping stiffness. Some authors (An and Guan,2004) compared quasi-static tire enveloping experiments under two tire pressures and three small-sized bumps and found that the static and quasi-static enveloping characteristics were similar, but there is a little difference in model validation. Presently, the simulation of tire mechanics by means of nonlinear finite element software can shorten the tire development cycle and can quickly simulate the mechanical performance of the tire under different working conditions. Furthermore, the position data that are not measured in the experiment can be obtained (Behroozinia and Khaleghian,2018; Sun et al.,2019).

There have been many detailed studies on the transverse, longitudinal, torsional, and radial stiffness, but there have been few studies on the enveloping stiffness of tires with different tire pressures, bump sizes and placement angle of bump under large load and deformation conditions. In this study, three different bump sizes, six different tire pressures, five different placement angles and large load were used to carry out multiple experiments on tires. Furthermore, establish models consider pressure and placement angle of bump, respectively, and establish a model consider pressure and placement angle simultaneously by cross-experiments. Using the Abaqus nonlinear finite element software to analysis stress and deformation distribution of tread and rebarforce of the skeleton ply under different bumps, and rebarforce of the skeleton ply with different placement angles.

\section{Methods}

Using the U-CAN UP-2092 experimental instrument, as shown in Fig.1 (a), the tire used is 195/65 R15. The room temperature was maintained at $24^{\circ} \mathrm{C}$, and the machine loading speed was set to $50 \mathrm{~mm} / \mathrm{min}$, because this study required a large load, and the experimental equipment was controlled by software, the damage energy was used as a uniform loading standard for the loading process. The tire damage energy calculation equation is as follows (Wei and Zhang,2017):

$$
\mathrm{W}=\mathrm{FD} / 2000
$$

where $\mathrm{W}$ is the damage energy, $\mathrm{F}$ is the load, and $\mathrm{D}$ is the vertical displacement.

The tire stiffness at a given pressure is derived from the displacement-load relationship over the normal working load range. The simplified equation for the tire stiffness can be expressed as follows:

$$
\mathrm{K}=\Delta \mathrm{F} / \Delta \mathrm{D}
$$

where $\Delta \mathrm{D}$ is the difference of vertical displacement, and $\Delta \mathrm{F}$ is the difference of load. Equation. (2) can also be differentiated using the numerical relationship between vertical displacement and load in the normal working load range, which is expressed as follows:

$$
\mathrm{K}=\mathrm{d}[\mathrm{F}(\mathrm{D})] / \mathrm{dD}
$$

where F(D) is vertical displacement-load function. Setting the loading energy in the instrument software and the loading was performed after the energy was set. Three different bump sizes were used in the experiment. The thicknesses of the bumps were 19, 32 and $38 \mathrm{~mm}$, and the diameter of the top half of the bumps were 19, 32 and 38 $\mathrm{mm}$, as shown in Fig. 1 (b). When performing the experiment under different size of bump, set four loading energy values: 200, 300, 350, and $400 \mathrm{~J}$, experiment according to Fig. 2 (a). When performing the experiment under different pressures, the bump size was fixed at $38 \mathrm{~mm}$ and set loading energy value as: $400 \mathrm{~J}$, different tire pressures were used 
in this experiment, from 0.16 to $0.31 \mathrm{MPa}$ by $0.03 \mathrm{MPa}$ increments. When performing the experiment with different placement angles of bump, the bump size was fixed at $38 \mathrm{~mm}$ and use $0.25 \mathrm{MPa}$ pressure, different angle of bump on platform were used in this experiment, from $0^{\circ}$ to $60^{\circ}$ by $15^{\circ}$ increments. Change the placement angle of bump as Fig. 2 (b).

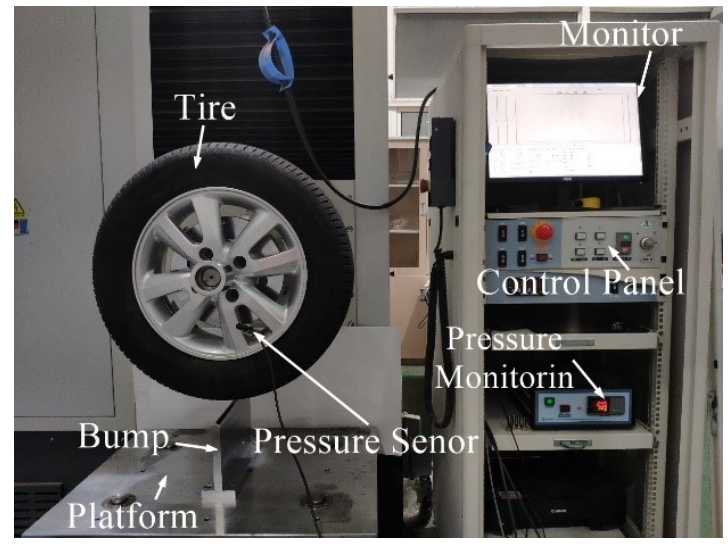

(a) Experimental instrument

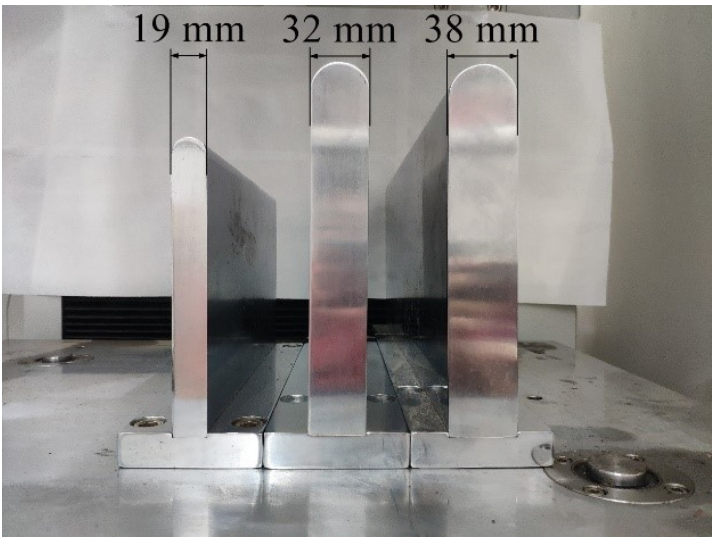

(b) Different size bumps

Fig. 1 UP-2092 experimental instrument and bumps.

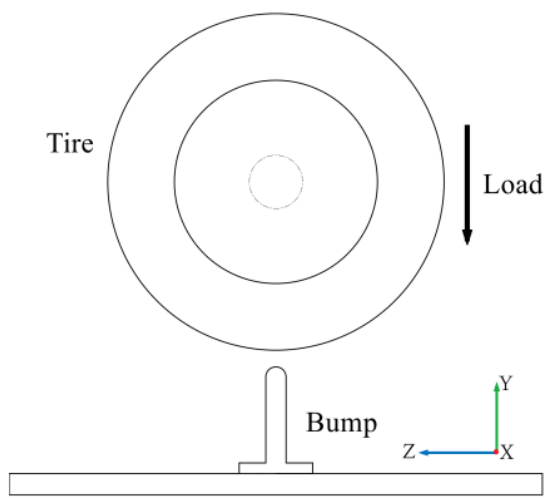

(a) Loading schematic

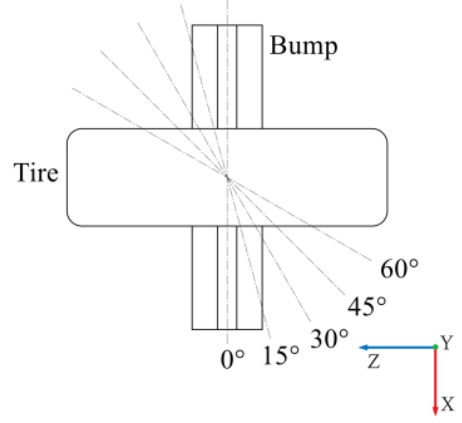

(b) Different placement angles of bump

Fig. 2 Experiment schematic diagrams.

\subsection{Experiment of enveloping stiffness with different bumps}

The vertical displacement-load curve and specific displacement and load values at different loading stages are shown in Fig. 3 and Table 1. It can be seen in Fig. 3 that the trends of the three displacement-load curves are basically the same, and they are not strictly linear. It can be seen in Table 1, the maximum vertical displacement in experiment was $80.0 \mathrm{~mm}, 79.37 \mathrm{~mm}, 78.93 \mathrm{~mm}$, and the corresponding maximum load was 10,002 N, 10,140 N, 10,258 N, respectively. Changes in maximum load and displacement during the experiment are less than $2 \%$. 


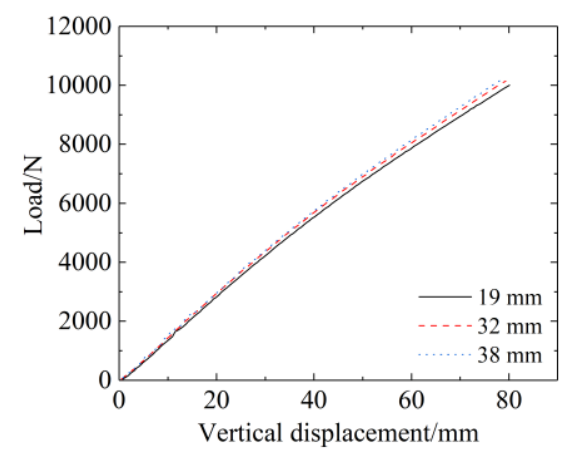

Fig. 3 Vertical displacement-load curve under different bumps.

Table 1 Value of displacement and load at different loading stages.

\begin{tabular}{cccccc}
\hline Bump size & & $200 \mathrm{~J}$ & $300 \mathrm{~J}$ & $350 \mathrm{~J}$ & $400 \mathrm{~J}$ \\
\hline \multirow{2}{*}{$19 \mathrm{~mm}$} & Displacement/mm & 54.77 & 68.26 & 74.44 & 80.08 \\
& Load/N & $7,330.1$ & $8,823.7$ & $9,432.9$ & 10,002 \\
\multirow{3}{*}{$32 \mathrm{~mm}$} & Displacement/mm & 54.23 & 67.58 & 73.54 & 79.37 \\
& Load/N & $7,389.1$ & $8,882.7$ & $9,550.8$ & 10,140 \\
& Displacement/mm & 54.21 & 67.13 & 73.31 & 78.93 \\
& Load/N & $7,467.7$ & $8,961.3$ & $9,570.5$ & 10,258 \\
\hline
\end{tabular}

\subsection{Experiment of enveloping stiffness under different pressures}

The vertical displacement-load curves are shown in Fig. 4. It can be found from Fig. 4 that under the same pressure, the load increases non-linearly with the increase of displacement. At $0.31 \mathrm{MPa}$, the load increases most rapidly with displacement, and at 0.16 and $0.19 \mathrm{MPa}$ the load increases most slowly with displacement, and the curve increased rapidly when the vertical displacement reached near 83.66 and $85.75 \mathrm{~mm}$, respectively. Because of the sidewalls were compressed to an almost folded state in the final stage of loading, and some internal surfaces of the sidewall had self-contacted. The self-contacting sidewall formed a whole piece of rubber, which improved the stiffness of the tire suddenly during this loading process.

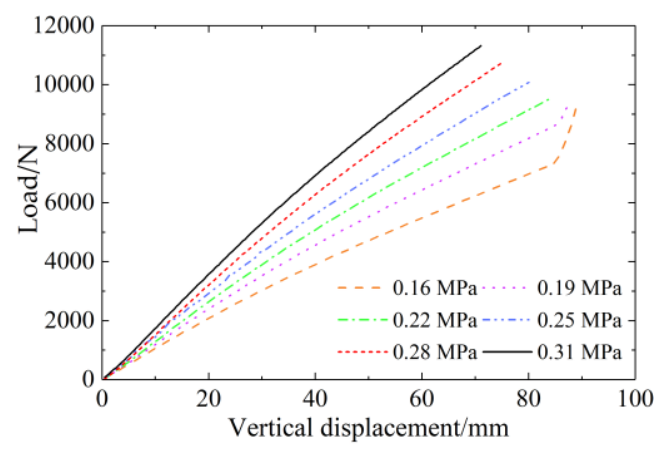

Fig. 4 Vertical displacement-load curve under different pressures.

The specific maximum displacement and load values under different pressure are shown in Table 2. With the increase of the tire pressure, the maximum displacement is continuously reduced, decreasing by $20.04 \%$, and the maximum load is continuously increasing, increasing by $22.80 \%$. The ratio of the decrease in the maximum displacement to the increase in the maximum load is close to 1, indicating that the effect of the increase in pressure on stiffness is almost linear. 
Table 2 Value of maximum displacement and load under different pressure.

\begin{tabular}{|c|c|c|c|c|c|c|}
\hline & \multicolumn{6}{|c|}{ Pressure/MPa } \\
\hline & 0.16 & 0.19 & 0.22 & 0.25 & 0.28 & 0.31 \\
\hline Displacement $_{\max } / \mathrm{mm}$ & 88.90 & 87.15 & 84.36 & 80.06 & 74.93 & 71.08 \\
\hline $\operatorname{Load}_{\max } / \mathrm{N}$ & $9,216.7$ & $9,216.7$ & $9,550.8$ & 10,081 & 10,729 & 11,319 \\
\hline
\end{tabular}

\subsection{Experiment of enveloping stiffness with different angle of bumps on platform}

The vertical displacement-load curves are shown in Fig. 5, and the value of maximum displacement and load are shown in Table 3. It can be seen in Fig. 5 that when the vertical displacement is less than $40 \mathrm{~mm}$, the growth trend of the displacement-load curve at each angle is basically the same, after the displacement exceeds $40 \mathrm{~mm}$, the difference between the each curves slowly increases as the angle increases, indicating that the change of the angle has little effect on the enveloping stiffness under a small vertical displacement. It can be seen from Table 3 that as the angle increases, the maximum vertical displacement decreases by $5.3 \%$ and the maximum load increases by $5 \%$, indicating that the impact of the change of the bump angle on the enveloping stiffness under large vertical displacement cannot be ignored.

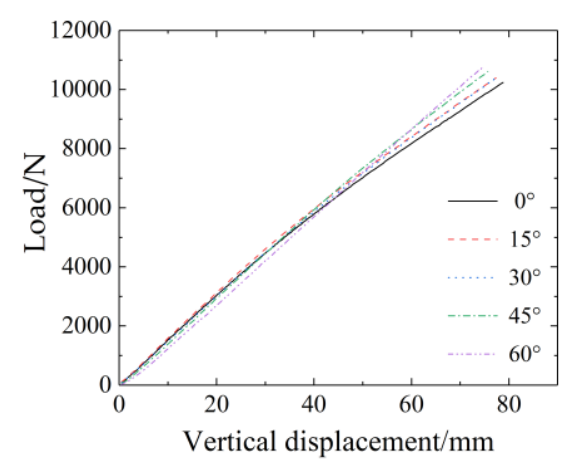

Fig. 5 Vertical displacement-load curve with different angles of bump.

Table 3 Value of maximum displacement and load with different angles of bump.

\begin{tabular}{cccccc}
\hline & \multicolumn{5}{c}{ Angle/ } \\
\cline { 2 - 6 } & 0 & 15 & 30 & 45 & 60 \\
\hline Displacement \\
Load $_{\text {max }} / \mathrm{N}$ & 78.77 & 77.37 & 77.37 & 75.69 & 74.58 \\
\hline
\end{tabular}

\subsection{Data analysis}

Use quartic polynomial without constant term to obtain the specific numerical relationship between the vertical displacement and enveloping stiffness force, multiple experiments were performed at different positions of the tire, and the displacement-load data (shown in Fig.3) was fitted to obtain a vertical displacement-load relationship as follow:

$$
F(D)=k_{1} D+k_{2} D^{2}+k_{3} D^{3}+k_{4} D^{4}
$$

Where $\mathrm{k}_{1}$ is linear stiffness coefficient, $\mathrm{k}_{2}, \mathrm{k}_{3}, \mathrm{k}_{4}$ are nonlinear stiffness coefficients, unit is $\mathrm{N} / \mathrm{mm}, \mathrm{N} / \mathrm{mm}^{2}, \mathrm{~N} / \mathrm{mm}^{3}$, $\mathrm{N} / \mathrm{mm}^{4}$. The coefficients under different sizes of bump were fitted by experiments data, the value of coefficients was shown in Table A1. The vertical displacement-enveloping stiffness relationship can be obtained from the first derivative of eq (4), as follows: 


$$
\begin{aligned}
& \mathrm{K}(\mathrm{D})=\mathrm{k}_{1}^{\prime}+\mathrm{k}_{2}^{\prime} \mathrm{D}+\mathrm{k}_{3}^{\prime} \mathrm{D}^{2}+\mathrm{k}_{4}^{\prime} \mathrm{D}^{3} \\
& \mathrm{k}_{1}^{\prime}=\mathrm{k}_{1} \\
& \mathrm{k}_{2}^{\prime}=2 \mathrm{k}_{2} \\
& \mathrm{k}_{3}^{\prime}=3 \mathrm{k}_{3} \\
& \mathrm{k}_{4}^{\prime}=4 \mathrm{k}_{4}
\end{aligned}
$$

Where $\mathrm{K}$ is enveloping stiffness, $\mathrm{k}_{1}{ }^{\prime}$ is linear stiffness coefficient, $\mathrm{k}_{2}^{\prime}, \mathrm{k}_{3}{ }^{\prime}, \mathrm{k}_{4}{ }^{\prime}$ are nonlinear stiffness coefficients, unit is $\mathrm{N} / \mathrm{mm}, \mathrm{N} / \mathrm{mm}^{2}, \mathrm{~N} / \mathrm{mm}^{3}, \mathrm{~N} / \mathrm{mm}^{4}$. It can be seen in Fig. 6 that the stiffness is basically the same when the displacement near $40 \mathrm{~mm}$, but the stiffness is different at the start and end loading positions. The maximum tire enveloping stiffness under different bump increased from 158.92 to $163.37 \mathrm{~N} / \mathrm{mm}$, increased by $2.80 \%$. And combined with Fig. 3, it can be considered that the change of the bump size has almost no effect on the enveloping stiffness.

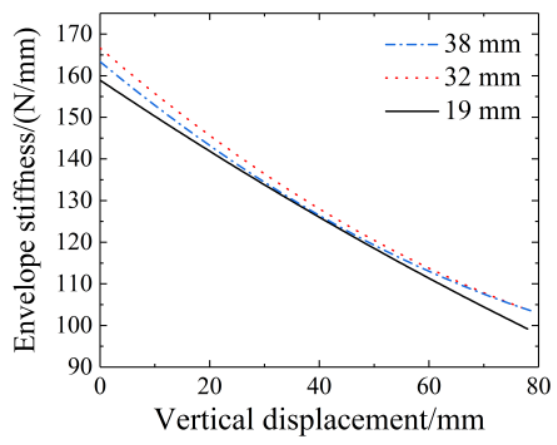

Fig. 6 Vertical displacement-enveloping stiffness curves under different size bumps.

The enveloping stiffness force models with $38 \mathrm{~mm}$ bump under different pressures can be obtained from Equation (6), and those stiffness coefficients can be obtained by fitting the experimental data (shown in Fig. 4). The value of stiffness coefficients shown in Table B1.

$$
F_{P}(D)=1_{1} D+1_{2} D^{2}+1_{3} D^{3}+1_{4} D^{4}
$$

The stiffness coefficient $l_{1}$ under different pressures can be obtained from Table B1 and shown by the stars in Fig. 7 (a), it can be seen that the stiffness coefficient $l_{1}$ increases linearly with the increase of pressure. In order to more accurately describe the change of the stiffness coefficient with pressure, a first-order polynomial is used for fitting, it shown by the line in Fig. 7 (a). The expressions of all fit coefficients are:

$$
\left\{\begin{array}{l}
1_{1}=a_{11}+a_{12} P \\
1_{2}=a_{21}+a_{22} P \\
1_{3}=a_{31}+a_{32} P \\
1_{4}=a_{41}+a_{42} P
\end{array}\right.
$$

Where $\mathrm{P}$ is pressure, MPa, $\mathrm{a}_{11}$ to $\mathrm{a}_{42}$ are pressure coefficients, it shown in Table B2. Bring (7) into (6), and (6) can be write as:

$$
F_{P}(D)=\left(a_{11}+a_{12} P\right) D+\left(a_{21}+a_{22} P\right) D^{2}+\left(a_{31}+a_{32} P\right) D^{3}+\left(a_{41}+a_{42} P\right) D^{4}
$$

Sorted out :

$$
F_{P}(D)=g(D)+P h(D)
$$


Where $g(D), h(D)$ are:

$$
\begin{aligned}
& g(D)=a_{11} D+a_{21} D^{2}+a_{31} D^{3}+a_{41} D^{4} \\
& h(D)=a_{12} D+a_{22} D^{2}+a_{32} D^{3}+a_{42} D^{4}
\end{aligned}
$$

Equation (8) is the enveloping stiffness force model considering tire pressure. Similarly, the model considering different placement angles of the bumps is Eq. (12), and the stiffness coefficients with $38 \mathrm{~mm}$ bump and $0.25 \mathrm{MPa}$ under different placement angles can be obtained by fitting the experimental data (shown in Fig. 5) are shown in Table C1.

$$
F_{A}(D)=j_{1} D+j_{2} D^{2}+j_{3} D^{3}+j_{4} D^{4}
$$

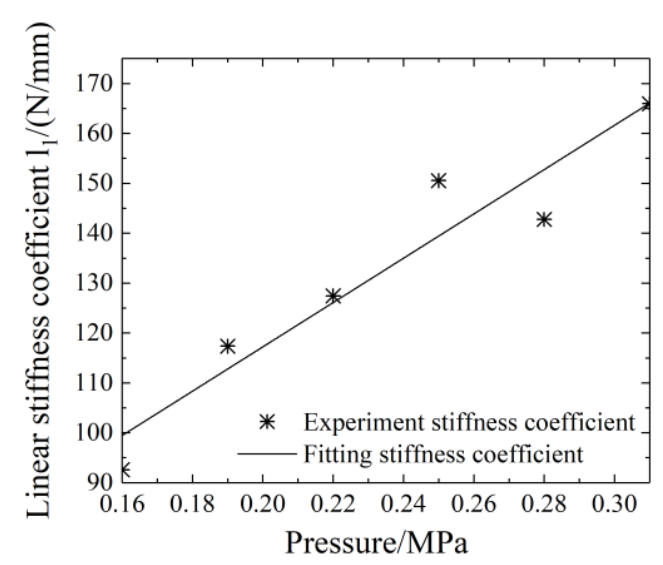

(a) $l_{1}$ under different pressures

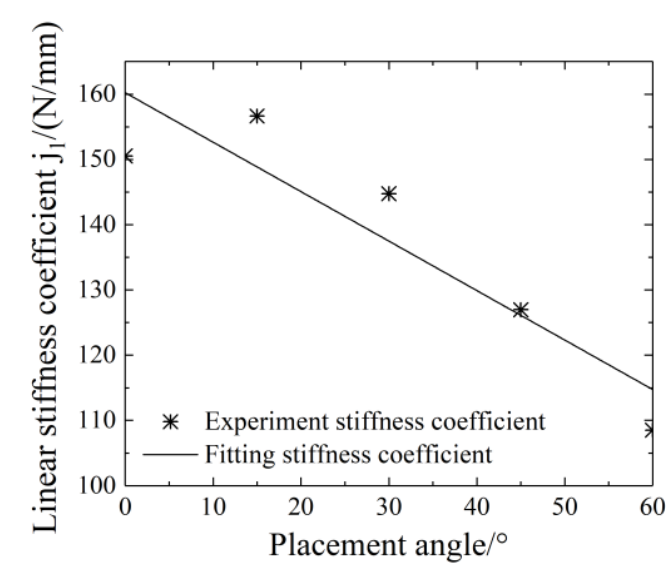

(b) $\mathrm{j}_{1}$ under different placement angles

Fig. 7 Change of linear stiffness coefficients under different condition.

The stiffness coefficient $\mathrm{j}_{1}$ of enveloping stiffness under different placement angles obtained from Table $\mathrm{C} 1$ are shown by the stars in Fig. 7 (b), it can be seen that the stiffness coefficient $\mathrm{j}_{1}$ decreases linearly with the increase of angles. The first-order polynomial can also be used for fitting the expressions of all coefficients, the expressions of $\mathrm{j}_{1}$ is shown by the line in Fig. 7 (b), and all the expressions are:

$$
\left\{\begin{array}{l}
\mathrm{j}_{1}=\mathrm{b}_{11}+\mathrm{b}_{12} \mathrm{~A} \\
\mathrm{j}_{2}=\mathrm{b}_{21}+\mathrm{b}_{22} \mathrm{~A} \\
\mathrm{j}_{3}=\mathrm{b}_{31}+\mathrm{b}_{32} A \\
\mathrm{j}_{4}=\mathrm{b}_{41}+\mathrm{b}_{42} A
\end{array}\right.
$$

Where $A$ is placement angel of bump, degree, $b_{11}$ to $b_{42}$ are angle coefficients, it shown in Table C2. Bring (13) into (12), Eq. (12) can be wrote as Eq. (14), and Eq. (14) is the enveloping stiffness force model considering placement angles of bump.

$$
F_{A}(D)=\left(b_{11}+b_{12} A\right) D+\left(b_{21}+b_{22} A\right) D^{2}+\left(b_{31}+b_{32} A\right) D^{3}+\left(b_{41}+b_{42} A\right) D^{4}
$$

Sorted out :

$$
F_{A}(D)=m(D)+A n(D)
$$

Where $\mathrm{m}(\mathrm{D}), \mathrm{n}(\mathrm{D})$ are: 


$$
\begin{aligned}
& m(D)=b_{11} D+b_{21} D^{2}+b_{31} D^{3}+b_{41} D^{4} \\
& n(D)=b_{12} D+b_{22} D^{2}+b_{32} D^{3}+b_{42} D^{4}
\end{aligned}
$$

The comparison of experiment value and calculated value maximum load by Eq. (8) and Eq. (14), it shown in Table 4 and 5. It can be seen in Table 4 that the errors are larger at 0.16 and $0.19 \mathrm{MPa}$, because the sudden change of load occurred in the final stage of the experiment, which has a greater impact on model fitting. Computed and experimental values agree well at $0.22-0.28 \mathrm{MPa}$ and large error at $0.31 \mathrm{MPa}$. According to Table 4, Eq. (8) has better predictability when the tire pressure is $0.19-0.28 \mathrm{MPa}$. In Table 5, the errors of computed and experimental values are both less than $10 \%$, indicating that Eq. (14) is in good agreement with the experiment.

Table 4 Maximum load by model and experiment under different pressures.

\begin{tabular}{cccccccc}
\hline & & \multicolumn{7}{c}{ Pressure/MPa } \\
\cline { 3 - 7 } & & 0.16 & 0.19 & 0.22 & 0.25 & 0.28 & 0.31 \\
\hline Load $_{\text {max }}$ & Model & $7,746.9$ & $8,558.8$ & $9,227.7$ & $9,669.4$ & $9,920.3$ & $10,232.6$ \\
$/ \mathrm{N}$ & Experiment & $9,216.7$ & $9,216.7$ & $9,550.8$ & 10,081 & 10,729 & 11,319 \\
& Error (\%) & 18.9 & 7.7 & 3.5 & 4.3 & 8.1 & 10.6 \\
\hline
\end{tabular}

Table 5 Maximum load by model and experiment under different placement angles.

\begin{tabular}{ccccccc}
\hline & & \multicolumn{5}{c}{ Angle ${ }^{\circ}$} \\
\cline { 3 - 7 } & & 0 & 15 & 30 & 45 & 60 \\
\hline Load $_{\max }$ & Model & $9,855.7$ & $10,352.4$ & $10,977.7$ & $11,309.6$ & $11,669.2$ \\
$/ \mathrm{N}$ & Experiment & 10,238 & 10,395 & 10,376 & 10,612 & 10,749 \\
& Error (\%) & 3.8 & 0.2 & 5.5 & 6.2 & 7.9 \\
\hline
\end{tabular}

Using a binary linear function as the objective function, Eq. (18), to establish a model consider pressure and placement angle of bump, and set $x$ and $y$ to $F_{A}(D)$ and $F_{P}(D)$, As shown in (19).

$$
\begin{aligned}
& f(x, y)=B x+C y+E \\
& F\left(F_{A}(D), F_{P}(D)\right)=B_{A}(D)+C_{P}(D)+E
\end{aligned}
$$

Cross experiment using six tire pressures and five placement angles to obtain 30 sets of displacement-load data, and use least squares (Wang and Liang,2013) to fit constants B, C, E in Eq. (19), set the weight of each data point to 1. The sum of error squares Q is:

$$
\begin{aligned}
& \mathrm{Q}(\mathrm{B}, \mathrm{C}, \mathrm{E})=\sum_{\mathrm{i}=1}^{\mathrm{n}}\left[\mathrm{z}_{\mathrm{i}}-\left(\mathrm{BF}_{\mathrm{A}}(\mathrm{D})+\mathrm{CF}_{\mathrm{P}}(\mathrm{D})+\mathrm{E}\right)\right]^{2} \\
& \mathrm{Q}(\mathrm{B}, \mathrm{C}, \mathrm{E})=\sum_{\mathrm{i}=1}^{\mathrm{n}}\left[\mathrm{z}_{\mathrm{i}}-\left(\mathrm{Bx}_{\mathrm{i}}+\mathrm{Cy}_{\mathrm{i}}+\mathrm{E}\right)\right]^{2}
\end{aligned}
$$

Where $z_{i}$ is experiment value of data, $x_{i}=F_{A}(D), y_{i}=F_{P}(D)$, Eq. (23) can be obtained from Eq. (22).

$$
\frac{\partial \mathrm{Q}}{\partial \mathrm{B}}=\frac{\partial \mathrm{Q}}{\partial \mathrm{C}}=\frac{\partial \mathrm{Q}}{\partial \mathrm{E}}=0
$$

Constants can be obtained by solving Eq. (22), and use C \# to program. Eq. (23) is an enveloping stiffness force calculation model considering pressure and placement angle of bump. The value of B, C, E shown in Table D1. 


$$
\mathrm{F}_{\mathrm{A}, \mathrm{P}}(\mathrm{D})=\mathrm{F}\left(\mathrm{F}_{\mathrm{A}}(\mathrm{D}), \mathrm{F}_{\mathrm{P}}(\mathrm{D})\right)=\mathrm{BF}_{\mathrm{A}}(\mathrm{D})+\mathrm{CF}_{\mathrm{P}}(\mathrm{D})+\mathrm{E}
$$

Randomly selected seven kinds of conditions using a model calculation, and compare with the experimental. It shown in Table 6, it can be seen from the table that the experiment values and calculated values are in good agreement. It shows that the Eq. (23) can calculate the tire enveloping stiffness force well under different conditions.

Table 6 Errors of enveloping stiffness force calculation model (Eq. (23)) under different conditions.

\begin{tabular}{|c|c|c|c|c|c|c|}
\hline \multicolumn{2}{|c|}{ Variables $\left(\mathrm{P} / \mathrm{MPa} / \mathrm{A} /{ }^{\circ} / \mathrm{D} / \mathrm{mm}\right)$} & \multicolumn{2}{|c|}{$0.16 / 0 / 88.9$} & \multicolumn{2}{|c|}{$0.22 / 15 / 17.03$} & $0.22 / 0 / 60.9$ \\
\hline \multirow{3}{*}{$\begin{array}{c}\text { Load } \\
\text { /N }\end{array}$} & Model & \multicolumn{2}{|c|}{$10,739.97$} & \multicolumn{2}{|l|}{$2,669.56$} & $8,333.27$ \\
\hline & Experiment & \multicolumn{2}{|l|}{9,217} & \multicolumn{2}{|l|}{2,339} & $7,271.2$ \\
\hline & Error $(\%)$ & \multicolumn{2}{|l|}{16.5} & \multicolumn{2}{|l|}{14.1} & 6.6 \\
\hline & \multicolumn{2}{|c|}{$0.25 / 60 / 18.77$} & \multicolumn{2}{|c|}{$0.28 / 60 / 28.96$} & \multicolumn{2}{|c|}{$0.31 / 45 / 21.24$} \\
\hline & \multicolumn{2}{|c|}{$2,738.31$} & \multicolumn{2}{|r|}{$4,389.51$} & \multicolumn{2}{|c|}{$3,286.68$} \\
\hline & \multicolumn{2}{|c|}{2,515} & \multicolumn{2}{|r|}{4,402} & \multicolumn{2}{|r|}{3,695} \\
\hline & \multicolumn{2}{|c|}{8.9} & & 0.2 & \multicolumn{2}{|r|}{12.4} \\
\hline
\end{tabular}

\section{Tire finite element analysis}

The Abaqus software was used to establish a finite element model of the tire and bump. The tire materials in the cross-section included the tread, sidewall, bead rubber, inner liner, belt rubber, carcass layer, rimcont and bead cords, as shown in Fig. 8. The carcass rubber and bead were established using deformable entities. The belt layers and carcass layer were established by surface entities, and use rebar unit. The bumps and wheels were defined as analytical rigid bodies. In the model, only the longitudinal pattern of the tread was established, and a single layer of the skeleton material was used to establish the belt layers and the carcass layer. The model of all rubber material is Neo Hooke, the carcass rubber and bead elements type were C3D8R, and use enhanced hourglass control to avoid element distortion. The belt and the carcass layers elements types were SFM3D4R, the orientation angle of belt 1 is $68^{\circ}$, belt 2 is $-68^{\circ}$ and carcass layer is $0^{\circ}$. The finite element model, as shown in Fig. 8, contained 39,060 elements for the carcass rubber and 21,430 elements in the belt and the carcass layers. The tread surface was set to contact the surface of the bump at the initial step, the friction coefficient of bump-tread surface is 0.4 and rim-rimcont surface is 0.8 , and add contact control to interactions to improve model convergence. At first step, all the degrees of freedom of the hub in the $\mathrm{X}$ and $\mathrm{Z}$ axes of the bump were fixed and exerted different pressures on inner liner surface. Set implicit procedure during the loading step, and the bump moved vertically along the $\mathrm{Y}$ axis.
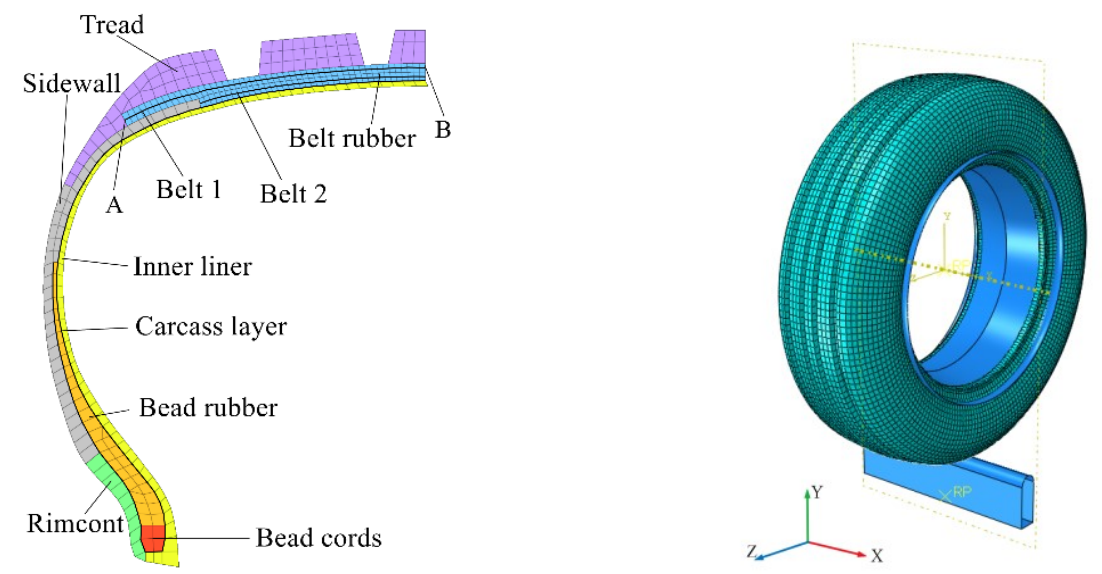

Fig. 8 The tire sectional structure (A, B are points of circumferential analysis on cords) and the finite element model for the tire and bump in Abaqus. 
Only the most common displacement conditions, from 0 to $45 \mathrm{~mm}$, in actual conditions were simulated. And because the time increment step was different during the calculation, the data at the time of the $45 \mathrm{~mm}$ displacement could not be accurately extracted when the result was extracted in the post-processing. Thus, the displacement when extracting the data was $45 \pm 2 \mathrm{~mm}$. The displacements of different bumps along the $\mathrm{Y}$ axis and the support reaction force (RF) from the simulation results were extracted and compared to the experimental data, as shown in Fig. 9. The difference between the simulated and experimental values were small, and under $32 \mathrm{~mm}$ bump, because of continuously random changing time increment steps, the result of simulation has some oscillation. However, the trend of three simulated curves was basically consistent with the experimental curve, which proved that the finite element model used in this paper was effective.

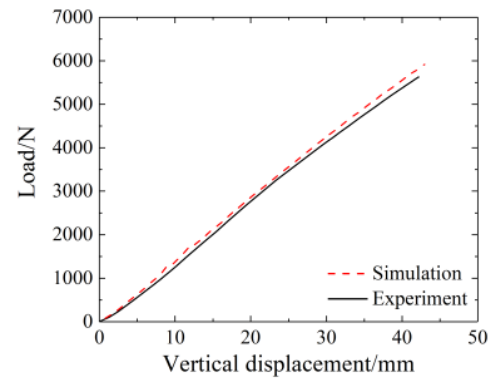

(a) $19 \mathrm{~mm}$ bump

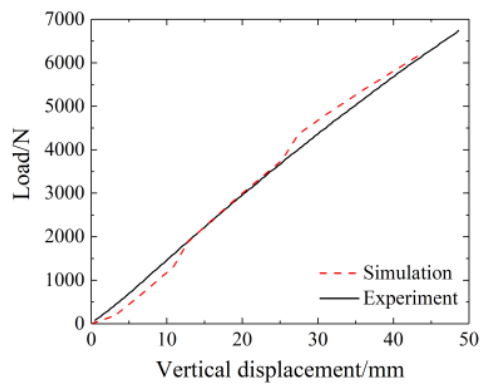

(b) $32 \mathrm{~mm}$ bump

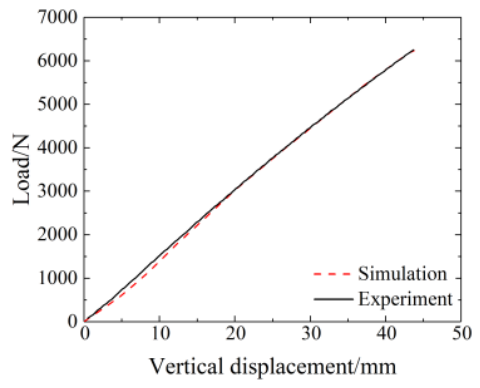

(c) $38 \mathrm{~mm}$ bump

Fig. 9 Comparison of experimental and simulated vertical displacement-load curves.

\subsection{Simulation of tread under different bumps}

It can be seen from the tread stress contours in Fig. 10, the stress distribution in the tread is H-shaped, the stress is greater on both sides of the tread, but in the center region of the tread is smaller, and the maximum stress value appears near the first groove on both sides of the tread. It can be seen from the tread stress contours, the deformation distribution in the tread is oval, the maximum value of deformation appears in center of tread. Combining the stress and deformation distribution of tread, it can be known that in the enveloping experiment, the stiffness of the center region of the tread is small, while the stiffness of both sides of the tread is large. The pressure distribution in the tread shown in Fig. 11, it can be seen in Fig. 11 that the pressure distribution of the three treads is basically the same, however the maximum pressure increases with the bump size decreases. Due to the reduction of bumps size, the contact area between the tread and the bumps is also constantly decreasing, resulting in acreage of the area where the maximum pressure appear is also decreasing. 

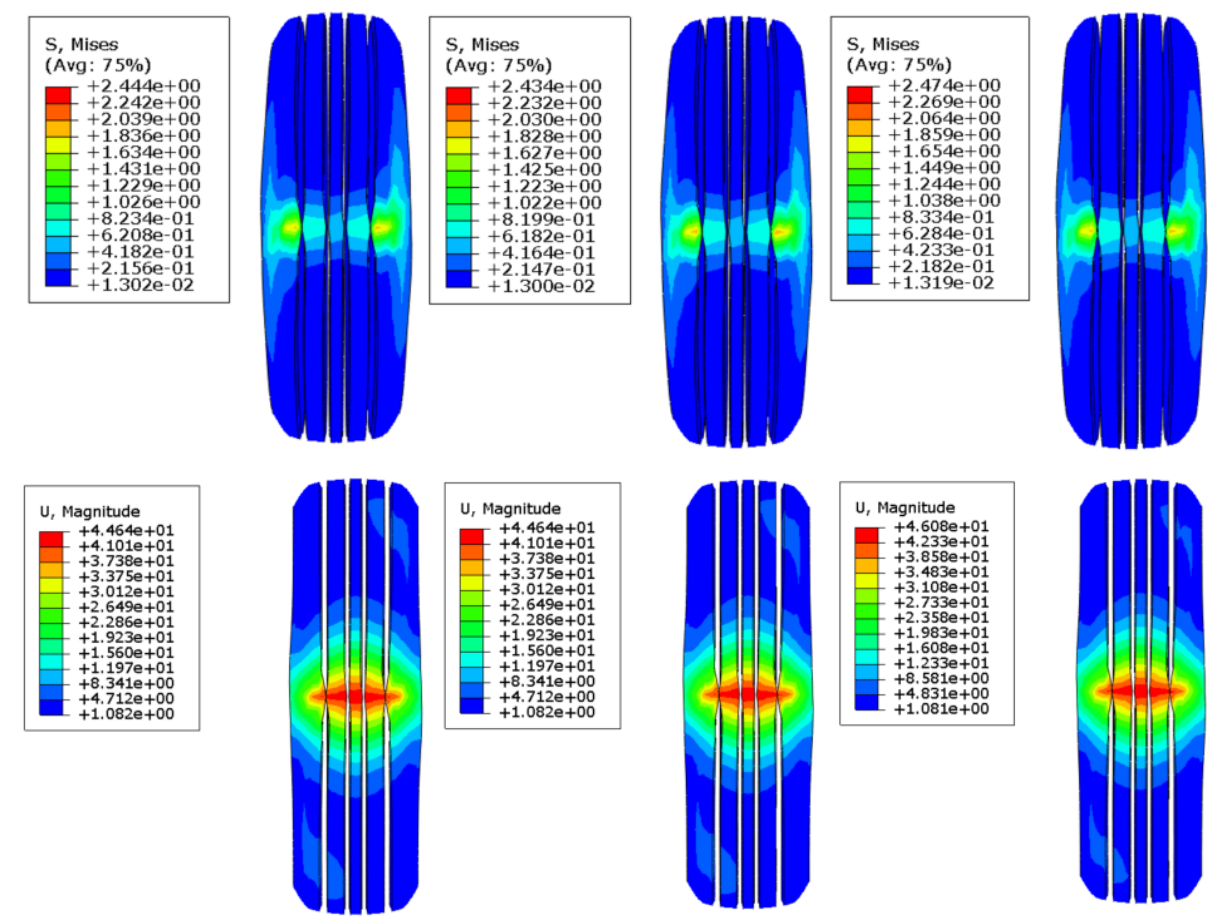

(b) $32-\mathrm{mm}$

(a) $38-\mathrm{mm}$

Fig. 10 Stress and deformation contours of tread under different bumps

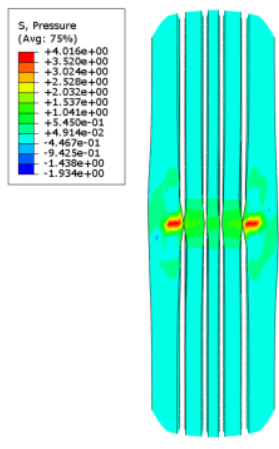

(a) $38-\mathrm{mm}$

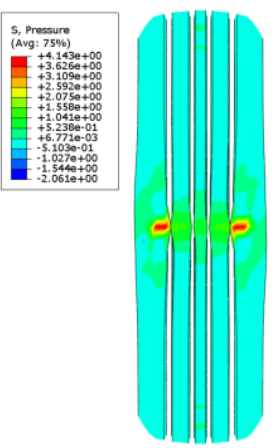

(b) $32-\mathrm{mm}$

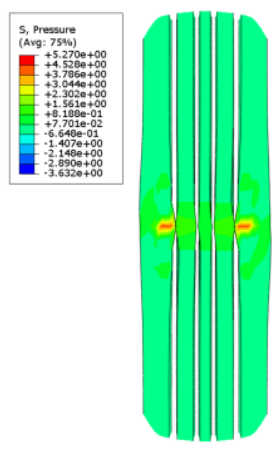

(c) $19-\mathrm{mm}$

Fig. 11 Pressure contours of tread under different bumps.

The rebarforce distribution of the belt 1 and belt 2 is symmetrical, as shown in Fig. 12 (a) and (b), the extreme value appears between the red dotted lines, and the direction is approximately the same as the direction of the cords, the minimum force value appears in area A shown in Fig. 13(a), and the value of rebarforce increase along the black arrow from the area A to other area, and the minimum force value appears in area B, indicating that belt failure may occur in this area during the enveloping process. Taking the bump as the dividing line (black line in the Fig. 13(a)), the length of the cord included in the area $\mathrm{A}$ is longer than in the area $\mathrm{B}$, and under the same loading displacement, the rebarforce of the shorter cord portion is larger, therefore area B is the area where the rebarforce is the largest, and because the force rebarforce in the area B is the largest, a lateral displacement occurs at the edge of the cord near area B. As shown in Fig. 12 (c), the force on both sides of the area in contact with the bump is small, and there are four symmetrical areas of small force. A shown in Fig. 13(b), because the bump is parallel to the cord, the rebarforce in the deformation area is small, in the $\mathrm{C}$ area, especially, is the smallest, and rebarforce continuously increases in the direction of the black arrow. After analysis the rebarforce distribution of ply, the " $\mathrm{H}$ " type stress distribution of the tread can be considered as the superposition of the force distribution of the three-layer skeleton. In addition, a large force value appeared on both sides of the three-layer cord of the contact area with the bump, and the force value was small in the center area of the contact. Comparing the distribution of the tread deformation, it can be seen that in the area where the rebarforce value is small, the tread deformation is large, and the tread deformation is small in the area where the rebarforce value is 
large, indicating that the force of the cord will directly affect the tire's enveloping stiffness.

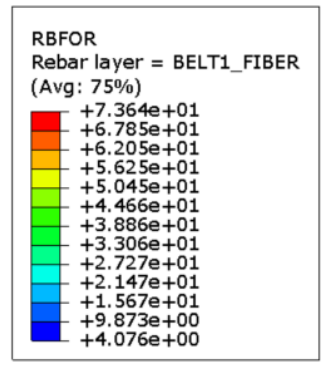

(a) belt 1

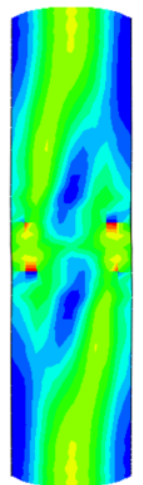

(b) belt 2
bump.

Fig. 12 Rebarforce contours of the ply under $38 \mathrm{~mm}$ bump.

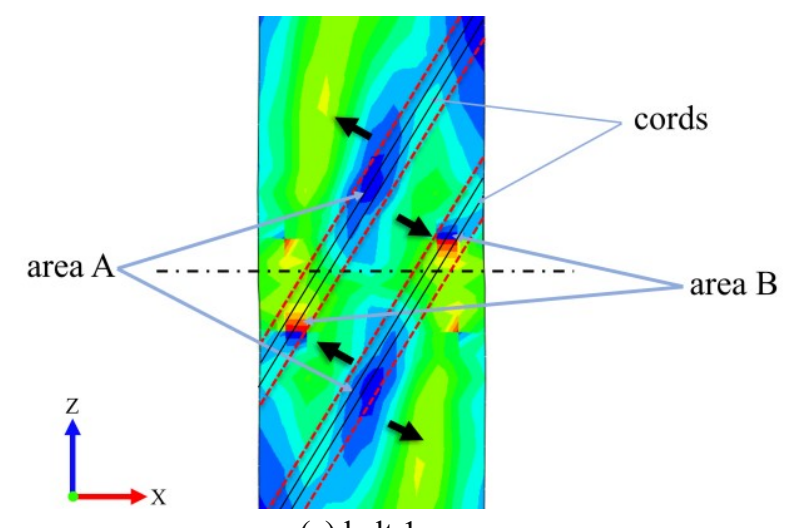

(a) belt 1

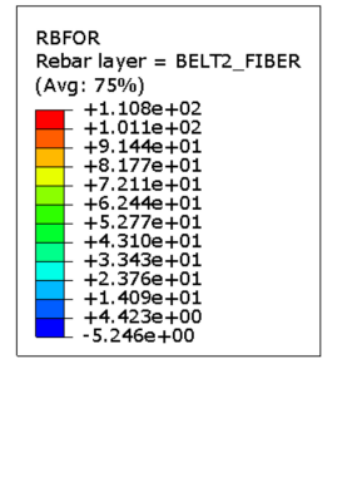

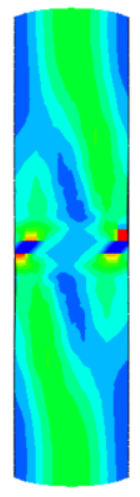

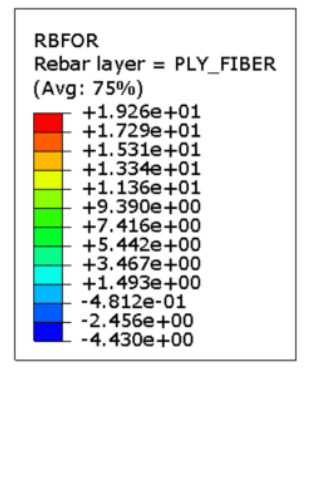

(c) carcass layer

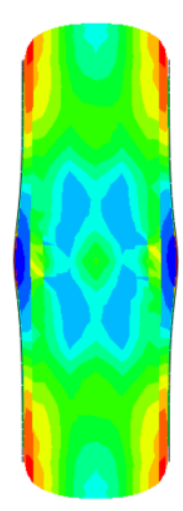

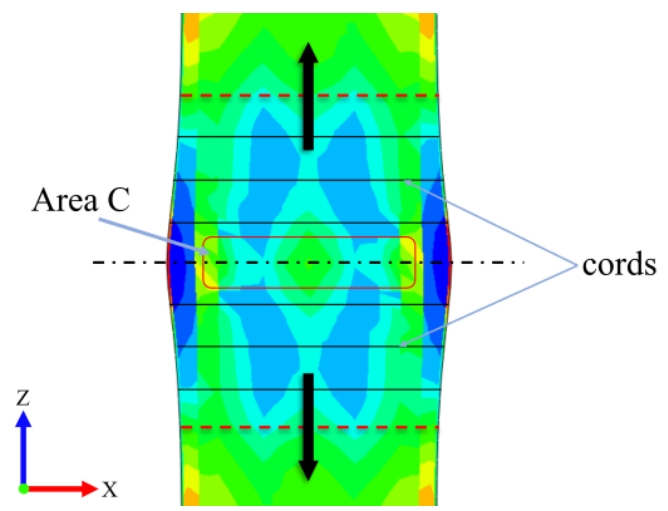

(b) carcass layer

Fig. 13 Areas with different force values and the direction of cords.

\subsection{Simulation of rebarforce with different placement angles of bump}

The rebarforce distribution of the belt 1 under different placement angles can be seen from Fig. 14. The area where the maximum rebarforce is constantly changing with the angle change, a large rebarforce appears at the upper and lower ends of the non-contact area along the angle of the cord ply, and as the angle increases, the ends of the area where the belt contacts the bumps are staggered(indicated by the black arrow in Fig. 14), and the maximum lateral displacement increased linearly with the angle of bump as shown in Fig. 15. The change trend of $0^{\circ}$ and $45^{\circ}$ is the same, and the maximum rebarforce appears on the left and right sides of the contact area with the bump, indicating that the cord is prone to failure in the contact deformation area when the bump is $0^{\circ}$ and $45^{\circ}$. The change trends of $15^{\circ}, 30^{\circ}$ and $60^{\circ}$ are the same, the maximum values of the rebarforce are less than $0^{\circ}$ and $45^{\circ}$, and when the angle is $60^{\circ}$, the distribution of force is similar to the angle of the cord ply, and the value of the rebarforce is the smallest in five groups. 


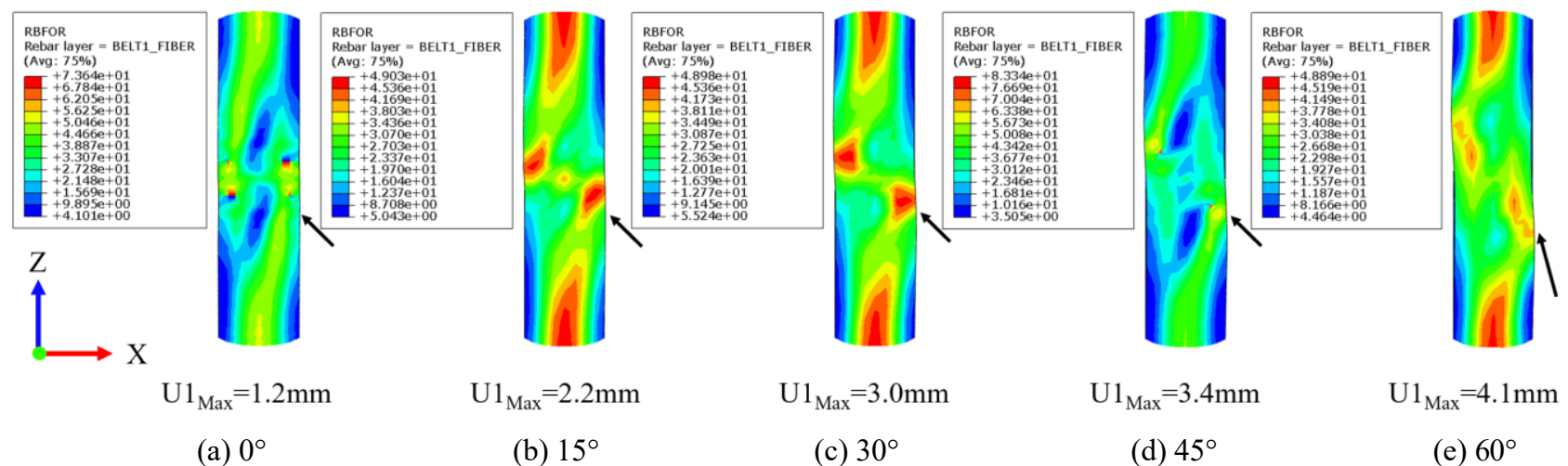

Fig. 14 Rebarforce contours of Belt 1 under different placement angles.

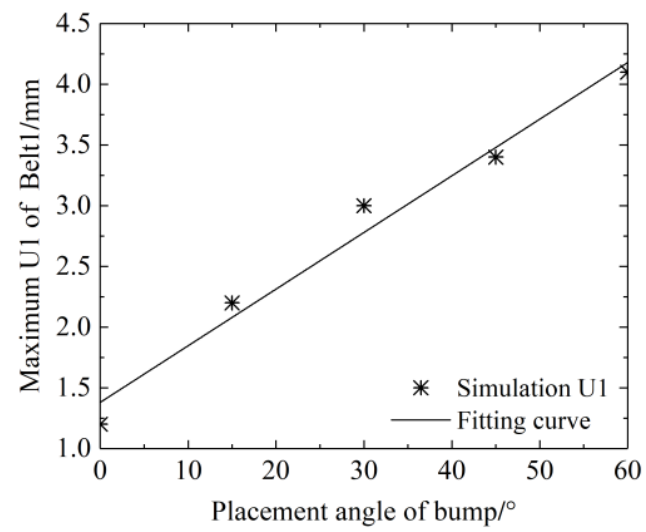

Fig. 15 Maximum lateral displacement(U1) of Belt 1 under different placement angles.

It can be seen in Fig. 16 (a) that the rebarforce of $0^{\circ}$ and $45^{\circ}$ is larger than others, and the max value of rebarforce appeared in the area contact with bump. Therefore, the end of belt should be strengthened. And it can be seen in Fig. 16 (b), the force in the middle of belt is small, and the force near middle area change suddenly, indicated the cord in this area prone to failure.

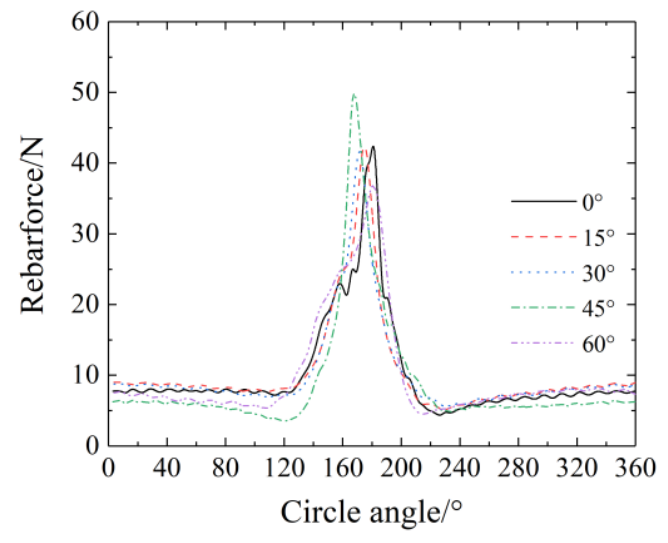

(a) Circumferential force on the cord end of Belt 1

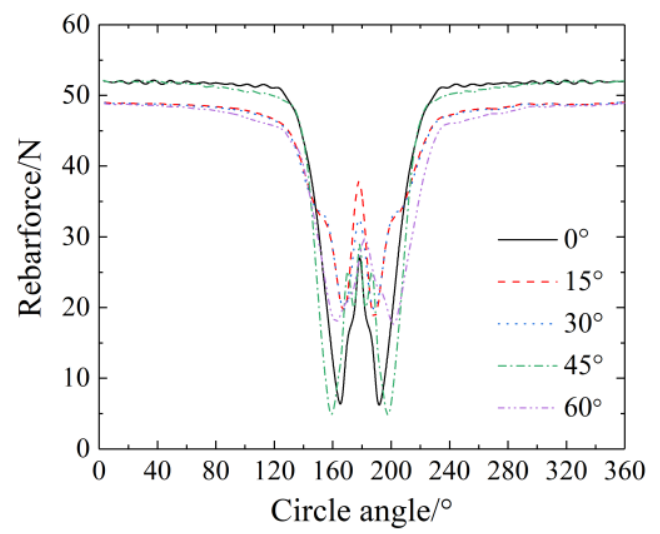

(b) Circumferential force on the cord middle of Belt 1

Fig. 16 Circumferential distribution of carcass cord force at different placement angles. The cord end of Belt 1 is point A in

Fig. 8. The cord middle of Belt 1 is point B in Fig. 8.

\section{Conclusions}

Three different bump sizes of 19, 32 and $38 \mathrm{~mm}$ were used to perform enveloping stiffness experiments to obtain the vertical displacement-load relationship of tires. The same experiment for different tire positions was used to fit the 
specific numerical relationship between vertical displacement and load, and the first derivative was computed to obtain the relationship between the vertical displacement and enveloping stiffness. A bump size of $38 \mathrm{~mm}$, six different tire pressures of $0.16,0.19,0.22,0.25,0.28,0.31 \mathrm{MPa}$ and five different placement angles $0^{\circ}, 15^{\circ}, 30^{\circ}, 45^{\circ}, 60^{\circ}$ were used to obtain the relationships between the vertical displacement and load, and establish models to calculate enveloping stiffness force consider pressure and placement angle of bump, respectively. Furthermore, establish a model to calculate enveloping stiffness force consider pressure and placement angle simultaneously by least squares. Use Abaqus to analysis the stress and deformation of tread and force of cord in ply. The following results were obtained:

(1). The bump size increased from 19 to $38 \mathrm{~mm}$, changes in maximum load and displacement during the experiment are less than $2 \%$, and the maximum values of the enveloping stiffness increased by $2.80 \%$. The bumps of different sizes had basically no effect on the tire enveloping stiffness under the same tire pressure.

(2). As the pressure increased from 0.16 to $0.31 \mathrm{MPa}$, the maximum load increased by $22.81 \%$ and the maximum vertical displacement decreased by $20.05 \%$, and sudden load changes under 0.16 and $0.19 \mathrm{MPa}$ due to self-contact of the sidewall in the final stage of loading. The ratio of the decrease in the maximum displacement to the increase in the maximum load is close to 1 , indicating that the effect of the increase in pressure on stiffness is almost linear. As the placement angle increases, the maximum vertical displacement decreases by $5.3 \%$ and the maximum load increases by $5 \%$, indicating that the effect of angle change cannot be ignore.

(3). The model calculates enveloping stiffness force consider pressure agree well at $0.19-0.28 \mathrm{MPa}$ but large error at 0.16 and $0.31 \mathrm{MPa}$. Therefore, this model has better predictability when the tire pressure is $0.19-0.28 \mathrm{MPa}$. The errors of model calculate enveloping stiffness force consider placement angle of bump are both less than $10 \%$, indicating that model is in good agreement with the experiment and has better predictability. The model consider pressure and angle simultaneously also has better predictability in enveloping stiffness force calculation, and this model allows enveloping stiffness force to be calculated without experiment.

(4). The "H" type stress distribution of the tread can be considered as the superposition of the force distribution of the three-layer skeleton, and the stress value in center region of the tread is small, and the maximum stress value appears near the first groove on both sides of the tread. The deformation distribution in the tread is oval, the maximum value of deformation appears in center of tread. Therefore, the enveloping stiffness in center region of the tread is small and in the both sides of the tread is maximum.

(5). The end of cord in belt is prone to failure in the contact deformation area when the bump is $0^{\circ}$ and $45^{\circ}$, and the cord near the middle of belt is prone to fracture. The change trends of $15^{\circ}, 30^{\circ}$ and $60^{\circ}$ are the same and the maximum values of the rebarforce are less than $0^{\circ}$ and $45^{\circ}$. When the angle is $60^{\circ}$, the distribution of rebarforce is similar to the angle of the cord ply, and the value of the rebarforce is the smallest. The force of the cord is greatly affected by the angle of the bump.

\section{Acknowledgments}

This entire study was supported by the National Natural Science Foundation of China (no. 51475399), Education and Scientific Research Projects for Middle and Young-Aged Teachers of Fujian (no.JAT190663) ,Science \& Technology Innovation Project of Fujian Province, China (no.2016H2003) and Research on the Impact of Tire Dynamics on the Handling and Stability of a Passenger Car (no.YKJCX2019012).

\section{Conflicts of Interest}

The authors declare that there are no conflicts of interest regarding the publication of the article. 


\section{Appendix A}

Table A1 Value of stiffness coefficient $\mathrm{k}$ under different size bumps.

\begin{tabular}{cccc}
\hline & \multicolumn{3}{c}{ Size of bump/mm } \\
\cline { 2 - 4 } & 19 & 32 & 38 \\
\hline $\mathrm{k}_{1} /(\mathrm{N} / \mathrm{mm})$ & 129.72 & 139.13 & 144.19 \\
$\mathrm{k}_{2} /\left(\mathrm{N} / \mathrm{mm}^{2}\right)$ & 0.964 & 0.74 & 0.5071 \\
$\mathrm{k}_{3} /\left(\mathrm{N} / \mathrm{mm}^{3}\right)$ & -0.0245 & -0.0222 & -0.0175 \\
$\mathrm{k}_{4} /\left(\mathrm{N} / \mathrm{mm}^{4}\right)$ & 0.0001 & 0.0001 & 0.0001 \\
\hline
\end{tabular}

\section{Appendix B}

Table B1 Value of stiffness coefficient 1 under different pressures.

\begin{tabular}{ccccccc}
\hline & \multicolumn{7}{c}{ Pressure/MPa } \\
\cline { 2 - 7 } & 0.16 & 0.19 & 0.22 & 0.25 & 0.28 & 0.31 \\
\hline $1_{1} /(\mathrm{N} / \mathrm{mm})$ & 92.58 & 117.36 & 127.42 & 150.53 & 142.75 & 165.96 \\
$1_{2} /\left(\mathrm{N} / \mathrm{mm}^{2}\right)$ & 1.094 & 0.3345 & 0.4608 & 0.292 & 1.4393 & 1.1352 \\
$1_{3} /\left(\mathrm{N} / \mathrm{mm}^{3}\right)$ & -0.0327 & -0.0142 & -0.0156 & -0.0148 & -0.036 & -0.0323 \\
$1_{4} /\left(\mathrm{N} / \mathrm{mm}^{4}\right)$ & 0.0002 & 0.0004 & 0.0001 & 0.0001 & 0.0002 & 0.0002 \\
\hline
\end{tabular}

Table B2 Value of pressure coefficients.

\begin{tabular}{cccc}
\hline $\mathrm{a}_{11} /(\mathrm{N} / \mathrm{mm})$ & 49.59 & $\mathrm{a}_{12} /(\mathrm{N} / \mathrm{mm} / \mathrm{MPa})$ & 366.17 \\
$\mathrm{a}_{21} /(\mathrm{N} / \mathrm{mm})$ & -0.4244 & $\mathrm{a}_{22} /(\mathrm{N} / \mathrm{mm} / \mathrm{MPa})$ & 0.2821 \\
$\mathrm{a}_{31} /(\mathrm{N} / \mathrm{mm})$ & 0.0022 & $\mathrm{a}_{32} /(\mathrm{N} / \mathrm{mm} / \mathrm{MPa})$ & -0.006 \\
$\mathrm{a}_{41} /(\mathrm{N} / \mathrm{mm})$ & $-0.0000,007$ & $\mathrm{a}_{42} /(\mathrm{N} / \mathrm{mm} / \mathrm{MPa})$ & $0.0000,3$ \\
\hline
\end{tabular}

\section{Appendix C}

Table C1 Value of stiffness coefficient $\mathrm{j}$ with different placement angles.

\begin{tabular}{lccccc}
\hline & \multicolumn{5}{c}{ Angle $/{ }^{\circ}$} \\
\cline { 2 - 6 } & 0 & 15 & 30 & 45 & 60 \\
\hline $\mathrm{j}_{1} /\left(\mathrm{N} / \mathrm{mm}^{2}\right)$ & 150.53 & 156.64 & 144.75 & 126.98 & 108.5 \\
$\mathrm{j}_{2} /\left(\mathrm{N} / \mathrm{mm}^{2}\right)$ & 0.292 & 0.2277 & 0.5884 & 1.4451 & 1.7853 \\
$\mathrm{j}_{3} /\left(\mathrm{N} / \mathrm{mm}^{3}\right)$ & -0.0148 & -0.0144 & -0.0179 & -0.0292 & -0.0298 \\
$\mathrm{j}_{4} /\left(\mathrm{N} / \mathrm{mm}^{4}\right)$ & 0.0001 & 0.0001 & 0.0001 & 0.0002 & 0.0002 \\
\hline
\end{tabular}

Table C2 Value of angle coefficients.

\begin{tabular}{cccc}
\hline $\mathrm{b}_{11} /(\mathrm{N} / \mathrm{mm})$ & 160.22 & $\mathrm{~b}_{12} /\left(\mathrm{N} / \mathrm{mm} /{ }^{\circ}\right)$ & -0.7581 \\
$\mathrm{~b}_{21} /(\mathrm{N} / \mathrm{mm})$ & 0.0269 & $\mathrm{~b}_{22} /\left(\mathrm{N} / \mathrm{mm} /{ }^{\circ}\right)$ & 0.028 \\
$\mathrm{~b}_{31} /(\mathrm{N} / \mathrm{mm})$ & -0.0123 & $\mathrm{~b}_{32} /\left(\mathrm{N} / \mathrm{mm} /{ }^{\circ}\right)$ & -0.0003 \\
$\mathrm{~b}_{41} /(\mathrm{N} / \mathrm{mm})$ & $0.0000,8$ & $\mathrm{~b}_{42} /\left(\mathrm{N} / \mathrm{mm} /{ }^{\circ}\right)$ & $0.0000,02$ \\
\hline
\end{tabular}

\section{Appendix D}

Table D1 Value of constants in Eq. (21).

\begin{tabular}{lc}
\hline $\mathrm{B}$ & $0.1920,3$ \\
$\mathrm{C}$ & $0.8503,4$ \\
$\mathrm{E}$ & $59.1182,4$ \\
\hline
\end{tabular}




\section{References}

An, H.W. and Guan, D.H. An experimental study on tire static enveloping properties. Automotive Engineering, Vol.26, No.1 (2004), pp.57-60.

Behroozinia, P. and Khaleghian, M. An investigation towards intelligent tyres using finite element analysis. International Journal of Pavement Engineering, (2018), pp.1-11.

Gent, A.N. and Walter, J.D. The Pneumatic Tire, (2013), pp.131-135 (in Chinese).

Guan, D.H. and Fan, C.J. Tire Modeling for Vertical Properties Including Enveloping Properties Using Experimental Modal Parameters. Vehicle System Dynamics: International Journal of Vehicle Mechanics and Mobility, Vol.40, No.6 (2003), pp.419-433.

Kang, N. Prediction of tire natural frequency with consideration of the enveloping property. International Journal of Automotive Technology, Vol.10, No.1 (2009), pp.65-71.

Karamihas, S.M. Alkana, V. and Anlas, G. Finite element modeling of static tire enveloping characteristics. International Journal of Automotive Technology, Vol.12, No.4 (2011), pp.529-535.

Li, X.H. Liu, C. and Lin, H. B. Finite element simulation of five-stiffness characteristics of tires. Rubber Technology, Vol.17, No.2 (2019), pp.21-25 (in Chinese).

Liu, Z. and Gao, Q. Analytical investigation on tire dynamics by rigid-elastic coupled tire model with nonlinear sidewall stiffness. Journal of the Brazilian Society of Mechanical Sciences and Engineering, Vol.40, No.2 (2018), p.80.

Mousseau, C.W. An Analytical and Experimental Study of a Tire Rolling Over a Stepped Obstacle at Low Velocity. Tire Science \& Technology, Vol.22, No.3 (1994), pp.162-181.

Qiu, X.D. Nonlinear characteristics of tire stiffness. Journal of Jilin University of Technology, No.4 (1994), pp.9-15 (in Chinese).

Shi, Q. Chen, W.W. and Hong, Y. Simulation study of tire stiffness characteristics based on finite element theory. Journal of System Simulation, Vol.18, No.6 (2006), pp.1445-1449 (in Chinese).

Sun, P.F. Huang, H.W and Zhou, S.T. Experiment and Analysis of Cord Stress on High-Speed Radial Tire Standing Waves. Shock and Vibration, Vol.2019, (2019), pp.9.

Veysel, A. Steven, M. and Gunay, A. Experimental analysis of tyre-enveloping characteristics at low speed. Vehicle System Dynamics, Vol.47, No.5 (2009), pp.575-587.

Wang, Q. Zhao, Y.Q. and Fu, H. Influences of loading and radial stiffness on mechanical elastic wheel enveloping characteristics. Transactions of the Chinese Society of Agricultural Engineering (2016).

Wang, G.L. and Liang, X.X. Research on Inverse Dynamics of Vehicle Handling Based on Tire Stability Matching. China Mechanical Engineering, Vol.243, No.3 (2013), pp.422-425 (in Chinese).

Wei, X.C. Zhang, X.F. and Zhang, Y.C. Car tire strength performance comparison analysis. Technology Information, Vol.15, No.31 (2017), pp.109-111 (in Chinese).

Ying, Z.F. and Zhou, T. Simulation analysis of automobile tire enveloping stiffness. China Automotive Engineering Society Annual Meeting (2009), (in Chinese).

Zou, L.Q. Finite element analysis of rolling transient collision of radial tire. Mechanical design and manufacturing, No.6 (2018), (in Chinese). 